\title{
Secretory response of the human pancreas to continuous intravenous infusion of secretin
}

\author{
J. G. BANWELL, B. E. NORTHAM, AND W. T. COOKE \\ From the General Hospital, Birmingham
}

EDITORIAL SYNOPSIS Measurement of the maximum secretory response of the pancreas is shown to be a valuable index of pancreatic function and the test is free from side effects.

Secretin has been prepared in a pure form with an activity of $17,500 \mathrm{u} . / \mathrm{mg}$. by Jorpes and Mutt (1964), and using this preparation, Baron, Perrier, Janowitz, and Dreiling (1963) and Hansky, Tiscornia, Dreiling, and Janowitz (1964) demonstrated a maximum pancreatic response in dogs for both volume and bicarbonate with continuous intravenous secretin infusion in doses varying from 4 to 16 units per minute. The present study was undertaken to measure the maximum secretory response of the human pancreas in normal subjects and in patients suspected of pancreatic disease and to compare these results with those of the standard secretin-pancreozymin tests described by Burton, Evans, Harper, Howat, Oleesky, Scott, and Varley (1960).

\section{MATERIAL}

NORMAL SUBJECTS Seven subjects, men and women aged 16 to 65 years of age, formed the control group. All had normal glucose tolerance tests, serum amylase levels, and faecal fat output, and were free of clinical and radiological evidence of pancreatic disease.

PATIENTS WITH BILIARY TRACT DISEASE Two patients (M.B. and M.L.) were studied following cholecystectomy, the pancreas appearing normal at laparotomy.

PATIENTS WITH PANCREATIC DISEASE Of four patients with chronic pancreatitis, two had steatorrhoea (H.E. and L.M.), one (C.A.) had pancreatic lithiasis, and all four had either latent or frank diabetes mellitus. In two (H.E. and L.M.) there was biopsy confirmation of chronic pancreatitis. One patient (M.H.) with an abnormal glucose tolerance test was suspected of having chronic relapsing pancreatitis on account of recurrent episodes of abdominal pain. One patient (D.C.) was studied three weeks after the onset of acute pancreatitis.

PATIENTS WITH MISCELLANEOUS DISORDERS

A group of six patients with miscellaneous disorders is shown in Figure $4 \mathrm{a}$ and $\mathrm{b}$. In all these patients, studies were arranged in the belief that pancreatic exocrine disease might be present

\section{METHODS}

All subjects were intubated after a 12-hour fast. A 14F Rehfus tube was positioned fluoroscopically to drain the descending and transverse portions of the duodenal loop and a 14 to 16 nasogastric tube was positioned to drain the body of the stomach. Both tubes were taped to the cheek to prevent forward movement. It was assumed that the position of the tubes had not altered significantly during the infusion since no change was demonstrated in the four patients whom it was convenient and possible to check. Throughout the test, subjects reclined in bed with head and shoulders comfortably raised on pillows. All patients lay towards the left side to facilitate pooling of gastric contents in the body of the stomach. Duodenal and gastric aspirates were collected separately in small flasks immersed in iced water. Aspiration was carried out using a Gomco thermotic drainage pump. The duodenal fluid was collected in 10-minute periods, the volume measured, and each specimen kept in iced water until analysed within a few hours of collection. The total volume of the duodenal Rehfus tube and connexion tubing to the flask was $9.5 \mathrm{ml}$.

The bicarbonate, bilirubin, amylase, and total protein concentrations were measured using methods adapted to the Technicon AutoAnalyzer (Northam and Banwell, 1966). The measurement of the bicarbonate content of duodenal fluid was based on the standard AutoAnalyzer method for plasma but using a more concentrated bicarbonate-carbonate buffer reagent to give a working range from 0 to $140 \mathrm{mEq}$. bicarbonate/ litre. To obtain an estimate of the bilirubin concentration, the duodenal fluid was diluted in the AutoAnalyzer with a bicarbonate-carbonate buffer at $p \mathrm{H} 8.5$ and the percentage transmission at $480 \mathrm{~m} \mu$ compared with that of bilirubin standards prepared from Dade bilirubin control 
serum. The amylase activity was measured using a modification of an automated amyloclastic method (Scheidt and Gochman, 1964).

Vitrum secretin was used throughout this study. Material from the same batch was used for any one individual study but different batches were used at different times during this work. Secretin was dissolved in normal saline and infused at rates varying from 1 to 18 $\mathrm{u}$./min. by a scalp vein needle into a hand vein using a Palmer constant infusion apparatus. Each infusion rate was maintained for 30 minutes. An interval of 10 minutes elapsed between the cessation of one dosage rate and the start of the next, the rate of infusion being increased stepwise. In eight subjects, a standard secretin-pancreozymin test (Burton et al., 1960) was performed immediately before the constant infusion of secretin.

\section{RESULTS}

BASAL COLLECTIONS IN NORMAL SUBJECTS The volume and bicarbonate output of the basal 10minute collections showed considerable variation from subject to subject (volume range 4 to $23 \mathrm{ml}$.: bicarbonate concentration 5 to $33 \mathrm{mEq}$./1.) These figures have not been analysed further. Spontaneous variation of the basal output has also been reported in dogs (Baron et al., 1963) and in man (Burton et al., 1960; Dreiling and Janowitz, 1962; Sarles, Bauer, and Prezlin, 1965).

CONTINUOUS INTRAVENOUS INFUSION IN NORMAL SUBJECTS Figure 1 illustrates the typical volume and bicarbonate response in a normal subject (M.P.) to stepwise increases in the rate of secretin infusion. The amylase and bilirubin outputs are also shown.

In general, the volume and bicarbonate response in the first 10 minutes of each 30 -minute infusion period was variable, the highest output being reached in the second and third 10-minute periods. The mean value of the second and third 10-minute collections was taken to represent the maximum response at each infusion rate. Figures 2 and 3 and Table I demonstrate the response to increasing secretin doses in the seven normal subjects. The maximum response was reached at secretin infusion rates of 4 to $6 \mathrm{u}$./ min., although in two subjects (M.K. and M.P.) a

TABLE I

'NORMAL' SUBJECTS

\begin{tabular}{|c|c|c|c|c|c|c|c|c|c|c|c|c|c|c|}
\hline \multirow[t]{4}{*}{ Patient } & \multirow[t]{4}{*}{ Diagnosis } & \multirow{4}{*}{$\begin{array}{l}\text { Body } \\
\text { Weight } \\
\text { (kg.) }\end{array}$} & \multirow{4}{*}{$\begin{array}{l}\text { Secretin } \\
\text { Dose } \\
\text { (units/ } \\
\text { min.) }\end{array}$} & \multicolumn{6}{|c|}{ Response } & \multicolumn{4}{|c|}{ Mean Response (Average of Samples 2 and 3) } & \multirow{4}{*}{$\begin{array}{l}\text { Maxi- } \\
\text { mum } \\
\text { Bicarbon- } \\
\text { ate Con- } \\
\text { centra- } \\
\text { tion } \\
(m E q . / 1 .)\end{array}$} \\
\hline & & & & \multirow{2}{*}{\multicolumn{3}{|c|}{$\begin{array}{l}\text { Volume (ml./ } \\
\text { min.): IO-Min. } \\
\text { Samples }\end{array}$}} & \multirow{2}{*}{\multicolumn{3}{|c|}{$\begin{array}{l}\text { Bicarbonate } \\
\text { ( } \mu \text { Eq./min.): } 10 \text { - } \\
\text { Min. Samples }\end{array}$}} & \multicolumn{2}{|l|}{ Volume } & \multicolumn{2}{|c|}{ Bicarbonate } & \\
\hline & & & & & & & & & & \multirow[t]{2}{*}{$\mathrm{ml} . / \mathrm{min}$} & \multirow[t]{2}{*}{$\mu l . / k g . / \min }$. & \multirow[t]{2}{*}{$\mu E q . / \min }$. & \multirow{2}{*}{$\begin{array}{l}\mu E q . / k g . / \\
\text { min. }\end{array}$} & \\
\hline & & & & $I$ & 2 & 3 & 1 & 2 & 3 & & & & & \\
\hline W.B. & $\begin{array}{l}\text { Colonic } \\
\text { diverticulitis }\end{array}$ & $71 \cdot 9$ & $\begin{array}{l}2 \\
4 \\
6\end{array}$ & $\begin{array}{l}4 \cdot 0 \\
3 \cdot 7 \\
8 \cdot 1\end{array}$ & $\begin{array}{l}4 \cdot 5 \\
5 \cdot 7 \\
5 \cdot 6\end{array}$ & $\begin{array}{l}4 \cdot 5 \\
6 \cdot 2 \\
4 \cdot 8\end{array}$ & $\begin{array}{l}356 \\
366 \\
738\end{array}$ & $\begin{array}{l}472 \\
621 \\
543\end{array}$ & $\begin{array}{l}531 \\
632 \\
470\end{array}$ & $\begin{array}{l}4 \cdot 5 \\
5 \cdot 9 \\
5 \cdot 2\end{array}$ & $\begin{array}{l}62 \\
82 \\
72\end{array}$ & $\begin{array}{l}501 \\
626 \\
506\end{array}$ & $\begin{array}{l}6 \cdot 96 \\
8 \cdot 70 \\
7 \cdot 03\end{array}$ & $\begin{array}{r}118 \\
102 \\
98\end{array}$ \\
\hline M.K. & Oesophagitis & $66 \cdot 1$ & $\begin{array}{l}1 \\
2 \\
4 \\
6\end{array}$ & $\begin{array}{l}1.0 \\
1.0 \\
2 \cdot 5 \\
3 \cdot 4\end{array}$ & $\begin{array}{l}1 \cdot 2 \\
2 \cdot 0 \\
5 \cdot 5 \\
5 \cdot 4\end{array}$ & $\begin{array}{l}0 \cdot 8 \\
3 \cdot 2 \\
5 \cdot 0 \\
5 \cdot 2\end{array}$ & $\begin{array}{r}15 \\
17 \\
298 \\
412\end{array}$ & $\begin{array}{r}23 \\
166 \\
705 \\
719\end{array}$ & $\begin{array}{r}17 \\
358 \\
505 \\
697\end{array}$ & $\begin{array}{l}1 \cdot 0 \\
2 \cdot 6 \\
5 \cdot 2 \\
5 \cdot 3\end{array}$ & $\begin{array}{l}15 \\
39 \\
79 \\
80\end{array}$ & $\begin{array}{r}20 \\
262 \\
605 \\
708\end{array}$ & $\begin{array}{r}0 \cdot 30 \\
3 \cdot 96 \\
9 \cdot 15 \\
10 \cdot 71\end{array}$ & $\begin{array}{r}21 \\
112 \\
128 \\
134\end{array}$ \\
\hline H.W. & Proctitis & $67 \cdot 9$ & $\begin{array}{l}1 \\
2 \\
4 \\
6\end{array}$ & $\begin{array}{l}3 \cdot 6 \\
2 \cdot 5 \\
4 \cdot 5 \\
2 \cdot 8\end{array}$ & $\begin{array}{l}3 \cdot 6 \\
4 \cdot 0 \\
5 \cdot 2 \\
5 \cdot 0\end{array}$ & $\begin{array}{l}3.8 \\
4.0 \\
5.0 \\
-\end{array}$ & $\begin{array}{l}360 \\
302 \\
490 \\
352\end{array}$ & $\begin{array}{l}417 \\
496 \\
598 \\
543\end{array}$ & $\begin{array}{l}463 \\
512 \\
570 \\
-\end{array}$ & $\begin{array}{l}3.7 \\
4.0 \\
5.1 \\
5.0\end{array}$ & $\begin{array}{l}54 \\
59 \\
75 \\
74\end{array}$ & $\begin{array}{l}440 \\
504 \\
584 \\
543\end{array}$ & $\begin{array}{l}6.47 \\
7 \cdot 40 \\
8 \cdot 60 \\
7 \cdot 99\end{array}$ & $\begin{array}{l}122 \\
128 \\
115 \\
126\end{array}$ \\
\hline P.W. & Epilepsy & $51 \cdot 3$ & $\begin{array}{r}2 \\
4 \\
6 \\
15\end{array}$ & $\begin{array}{l}1 \cdot 1 \\
3 \cdot 0 \\
2 \cdot 5 \\
4 \cdot 2\end{array}$ & $\begin{array}{l}1.5 \\
2.5 \\
4.0 \\
3.3\end{array}$ & $\begin{array}{l}3.0 \\
3.5 \\
3.5 \\
4.0\end{array}$ & $\begin{array}{r}97 \\
246 \\
282 \\
268\end{array}$ & $\begin{array}{l}147 \\
200 \\
484 \\
280\end{array}$ & $\begin{array}{l}246 \\
231 \\
406 \\
452\end{array}$ & $\begin{array}{l}2.2 \\
3.0 \\
3.7 \\
3.6\end{array}$ & $\begin{array}{l}43 \\
58 \\
72 \\
70\end{array}$ & $\begin{array}{l}197 \\
215 \\
446 \\
366\end{array}$ & $\begin{array}{l}3 \cdot 84 \\
4 \cdot 20 \\
8 \cdot 70 \\
7 \cdot 14\end{array}$ & $\begin{array}{r}88 \\
112 \\
121 \\
113\end{array}$ \\
\hline P.K. & $\begin{array}{l}\text { Glandular } \\
\text { tuberculosis }\end{array}$ & $49 \cdot 5$ & $\begin{array}{l}1 \\
2 \\
4 \\
6 \\
8\end{array}$ & $\begin{array}{l}0.3 \\
0.6 \\
0.3 \\
2.4 \\
3.4\end{array}$ & $\begin{array}{l}1.0 \\
1.5 \\
2.5 \\
3.4 \\
3.0\end{array}$ & $\begin{array}{l}1 \cdot 6 \\
1 \cdot 3 \\
2 \cdot 5 \\
2 \cdot 9 \\
3 \cdot 1\end{array}$ & $\begin{array}{r}5 \\
53 \\
18 \\
276 \\
404\end{array}$ & $\begin{array}{r}69 \\
157 \\
238 \\
384 \\
345\end{array}$ & $\begin{array}{l}134 \\
139 \\
310 \\
351 \\
362\end{array}$ & $\begin{array}{l}1 \cdot 3 \\
1 \cdot 4 \\
2 \cdot 5 \\
3 \cdot 1 \\
3 \cdot 0\end{array}$ & $\begin{array}{l}26 \\
28 \\
50 \\
63 \\
61\end{array}$ & $\begin{array}{l}101 \\
148 \\
274 \\
367 \\
353\end{array}$ & $\begin{array}{l}2 \cdot 04 \\
2.99 \\
5 \cdot 53 \\
7 \cdot 41 \\
7 \cdot 13\end{array}$ & $\begin{array}{r}84 \\
107 \\
124 \\
121 \\
117\end{array}$ \\
\hline M.P. & Proctitis & $70 \cdot 0$ & $\begin{array}{r}2 \\
4 \\
6 \\
10\end{array}$ & $\begin{array}{l}1 \cdot 2 \\
3 \cdot 5 \\
4 \cdot 7 \\
4 \cdot 5\end{array}$ & $\begin{array}{l}3.0 \\
3.8 \\
5.6 \\
5.0\end{array}$ & $\begin{array}{l}3.4 \\
3 \cdot 8 \\
4.8 \\
5 \cdot 0\end{array}$ & $\begin{array}{r}44 \\
350 \\
475 \\
491\end{array}$ & $\begin{array}{l}309 \\
421 \\
498 \\
510\end{array}$ & $\begin{array}{l}388 \\
426 \\
504 \\
510\end{array}$ & $\begin{array}{l}3 \cdot 2 \\
3 \cdot 8 \\
5 \cdot 2 \\
5 \cdot 0\end{array}$ & $\begin{array}{l}46 \\
54 \\
74 \\
71\end{array}$ & $\begin{array}{l}348 \\
423 \\
501 \\
510\end{array}$ & $\begin{array}{l}4 \cdot 98 \\
6 \cdot 04 \\
7 \cdot 16 \\
7 \cdot 28\end{array}$ & $\begin{array}{l}114 \\
113 \\
105 \\
102\end{array}$ \\
\hline F.W. & Psychoneurosis & 50.4 & $\begin{array}{l}2 \\
4 \\
6 \\
8\end{array}$ & $\begin{array}{l}1.5 \\
1.9 \\
1.5 \\
2.7\end{array}$ & $\begin{array}{l}1.9 \\
3.3 \\
3 \cdot 3 \\
3.7\end{array}$ & $\begin{array}{l}1 \cdot 9 \\
3 \cdot 3 \\
4 \cdot 0 \\
4 \cdot 1\end{array}$ & $\begin{array}{l}108 \\
250 \\
156 \\
253\end{array}$ & $\begin{array}{l}228 \\
366 \\
366 \\
398\end{array}$ & $\begin{array}{l}248 \\
346 \\
437 \\
387\end{array}$ & $\begin{array}{l}1.9 \\
3.3 \\
3.6 \\
3.9\end{array}$ & $\begin{array}{l}38 \\
65 \\
71 \\
77\end{array}$ & $\begin{array}{l}238 \\
356 \\
401 \\
393\end{array}$ & $\begin{array}{l}4 \cdot 73 \\
7 \cdot 07 \\
7 \cdot 95 \\
7 \cdot 80\end{array}$ & $\begin{array}{l}127 \\
111 \\
111 \\
106\end{array}$ \\
\hline
\end{tabular}




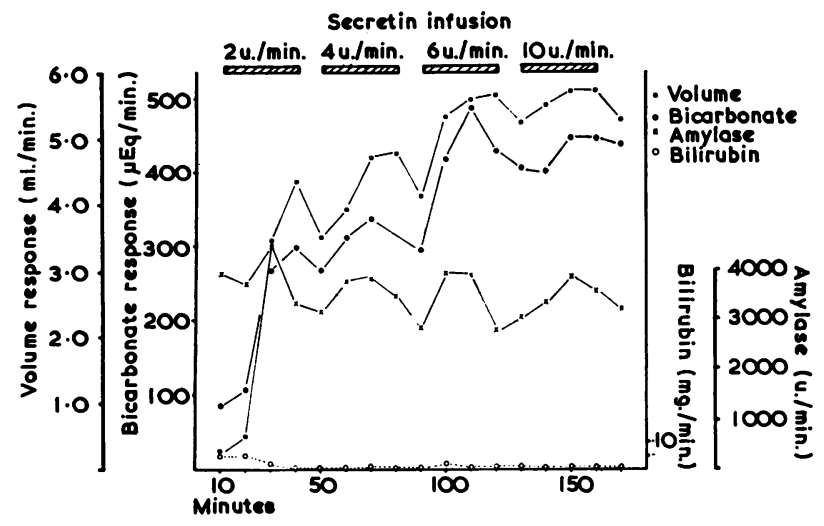

FIG. 1. The characteristic response to progressive increase in the rate of secretin infusion in a normal subject (M.P.).

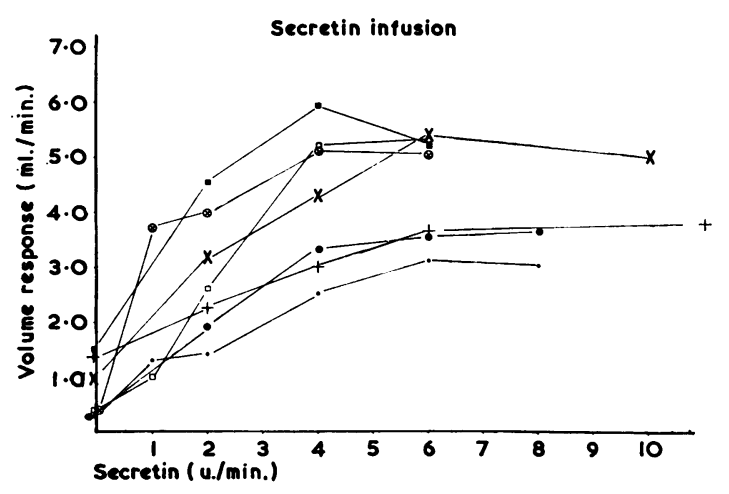

FIG. 2. The volume response to progressive increase in the rate of secretin infusion in seven normal subjects.

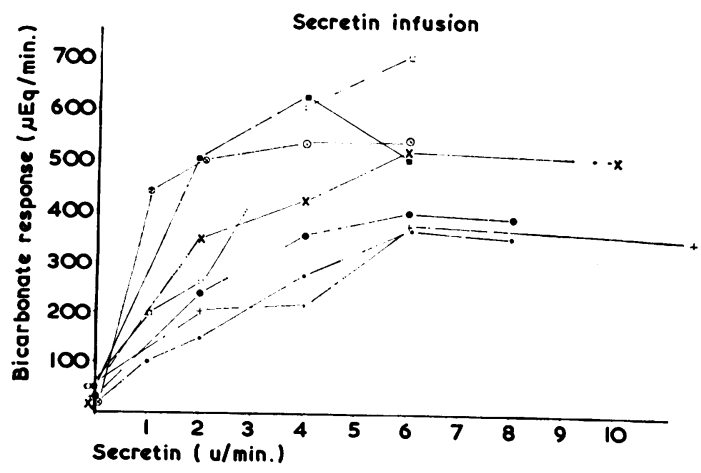

FIG. 3. The bicarbonate response to progressive increase in the rate of secretin infusion in seven normal subjects. maximum response was not clearly attained and it may have been submaximal. The maximum bicarbonate concentration ranged from 118 to $134 \mathrm{mEq}$./1., and in five subjects was reached before a maximum secretory response for volume and bicarbonate output had been attained. There was a tendency for the bicarbonate concentration to fall with supramaximum doses and when infusion was prolonged to more than 100 minutes.

Bilirubin concentration in the duodenal fluid was low throughout most infusions.

The amylase output in the duodenal fluid remained relatively constant with increasing rates of secretin infusion.

Relationship between maximum secretory response and body size The relationship between body weight and the maximum volume and bicarbonate response to secretin infusion in the seven normal subjects is shown in Figure $4 a$ and $b$. The regression of response on body weight (Table II) was found to be highly significant for the maximum volume response $(\mathrm{P}<0.001)$ and significant for the maximum bicarbonate response $(P=0.02)$. Similarly, there was a significant regression of response on surface area (calculated from Dubois tables).

GASTROINTESTINAL DISORDERS The maximum volume and bicarbonate response in various gastrointestinal disorders is shown in Figure $4 a$ and $b$ and Table III.

Biliary tract disease The volume response for both patients (M.L. and M.B.) was higher than for the normal subjects, though bicarbonate output was within the normal range. Burton and co-workers (1960) had noted a significant increase in the mean volume of duodenal contents in similar patients using the secretin pancreozymin test.

Chronic pancreatitis All patients had abnormally low maximum volume and bicarbonate responses. In 
TABLE II

REGRESSION (IF MAXIMUM VOLUME RESPONSE $\left(Y_{1}\right)$ AND MAXIMUM BICARBONATE RESPONSE $\left(Y_{2}\right)$ UPON BODY WEIGHT $(X)$ IN SEVEN NORMAL SUBJECTS

\begin{tabular}{|c|c|c|c|c|c|c|c|c|}
\hline & \multirow[b]{2}{*}{$\begin{array}{l}\text { Regression Equation } \\
Y=b X+a\end{array}$} & \multicolumn{3}{|c|}{ Test of Significance of Regression } & \multirow[b]{2}{*}{$\begin{array}{r}X \\
k g\end{array}$} & \multirow[b]{2}{*}{$\boldsymbol{Y}$} & \multicolumn{2}{|l|}{ Normal Range } \\
\hline & & S.E. (b) & $t=\frac{b}{S . E .(b)}$ & $\stackrel{P}{a t} n=5$ & & & $\begin{array}{l}\text { S.D. of a Single } \\
\text { Observation of } Y \\
\text { about Regression } \\
\text { Line at Given } \\
\text { Values of } X\end{array}$ & $\begin{array}{l}95 \% \text { Confidence } \\
\text { Limits for a Single } \\
\text { Observation of } Y \text { at } \\
\text { Given Values of } X \\
(n=5, t=2 \cdot 57)\end{array}$ \\
\hline $\begin{array}{l}Y_{1} \\
\text { Maximum volume } \\
\text { response (ml./min.) }\end{array}$ & $Y_{1}=0.098 X-1.379$ & 0.0123 & $7 \cdot 97$ & $<0.001$ & $\begin{array}{l}50 \\
61 \\
75\end{array}$ & $\begin{array}{l}3 \cdot 52 \\
4 \cdot 60 \\
5 \cdot 97\end{array}$ & $\begin{array}{l}0.351 \mathrm{ml} . / \mathrm{min} . \\
0.325 \mathrm{ml} . / \mathrm{min} . \\
0.367 \mathrm{ml} . / \mathrm{min}\end{array}$ & $\begin{array}{l} \pm 0.90 \mathrm{ml} . / \mathrm{min} \\
\pm 0.83 \mathrm{ml} . / \mathrm{min} \\
\pm 0.94 \mathrm{ml} . / \mathrm{min}\end{array}$ \\
\hline $\begin{array}{l}Y_{2} \\
\text { Maximum bicarbonate } \\
\text { response }(\mu \mathrm{Eq} . / \mathrm{min} .)\end{array}$ & $Y_{2}=10 \cdot 12 X-97 \cdot 25$ & $2 \cdot 915$ & 3.47 & 0.02 & $\begin{array}{l}50 \\
61 \\
75\end{array}$ & $\begin{array}{l}409 \\
520 \\
663\end{array}$ & $\begin{array}{l}91.28 \mu \mathrm{Eq} . / \mathrm{min} . \\
84.33 \mu \mathrm{Eq} . / \mathrm{min} . \\
95.46 \mu \mathrm{Eq} . / \mathrm{min} .\end{array}$ & $\begin{array}{l} \pm 235 \mu \mathrm{Eq} . / \mathrm{min} . \\
\pm 217 \mu \mathrm{Eq} . / \mathrm{min} . \\
\pm 245 \mu \mathrm{Eq} . / \mathrm{min} .\end{array}$ \\
\hline
\end{tabular}

(a) Maximum volume response

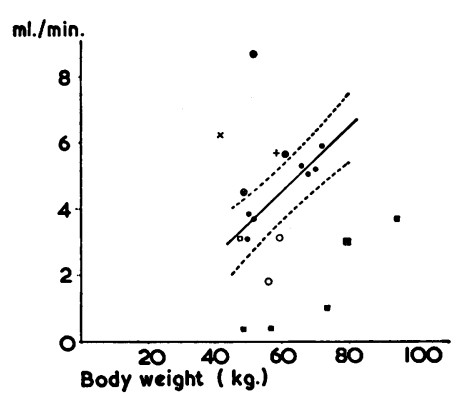

(b) Maximum bicarbonate response

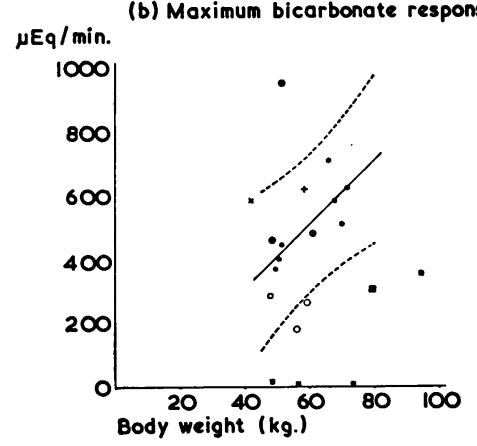

FIG. 4a. The maximum volume.

FIG. 4b. The maximum bicarbonate response of normal subjects and patients with gastrointestinal disorders related to body weight.

\footnotetext{
- Normal subjects.

- Chronic pancreatitis.

- Unproved chronic pancreatitis.

- Adult coeliac disease.

Q After cholecystectomy.

Diabetes mellitus.

+ Post acute pancreatitis.

$\times$ Idiopathic steatorrhoea.

$\oplus$ Diarrhoea, hyperchlorhydria.
}

three (L.M., H.E., and L.S.) the maximum bicarbonate concentration attained was low $(45,35$, and 20 mEq./l. respectively): in C.A. the bicarbonate concentration (96 mEq./1.) approached the normal level (123 \pm S.D. $6.8 \mathrm{mEq} . / 1$.$) .$

Suspected chronic pancreatitis The patient (M.H.) had maximum volume and bicarbonate output within the normal range.

Post-acute pancreatitis The patient (D.C.) had an increased total volume output but the bicarbonate level was within the normal range.

MISCELlaNeOus Disorders The two patients (B.G. and C.G.) with adult coeliac disease who were studied before treatment both had low maximum volume and bicarbonate responses. One patient (R.M.) with diabetes mellitus had abnormally low responses. Moderate impairment of pancreatic function has been recorded previously by Pollard, Miller, and Brewer (1943) and by Chey, Shay, and Shuman (1963) in primary diabetes mellitus.

Two patients, one with idiopathic steatorrhoea (D.T.) and the other (J.T.) with diarrhoea and increased gastric acid output (basal acid output $29 \cdot 4$ $\mathrm{mEq}$./hr. and maximum acid output of $48.0 \mathrm{mEq} . / \mathrm{hr}$.), had volume responses that greatly exceeded the normal range. J. T. also showed a markedly increased bicarbonate output and that of D.T. was at the upper limit of normal.

COMPARISON OF THE RESPONSE TO THE SECRETINPANCREOZYMIN TEST (BURTON TEST) AND TO MAXIMUM SECRETIN INFUSION Eight patients had both Burton tests and constant secretin infusions performed at maximum doses. Two were normal subjects: six had miscellaneous gastrointestinal disorders.

The Burton test gave an abnormal volume response for the two patients (L.M. and H.E.) with chronic pancreatitis, but normal results were obtained on the two normal subjects (W.B. and M.W.), on one patient after cholecystectomy (M.L.), and on 


\section{TABLE III}

MAXIMUM VOLUME AND BICARBONATE RESPONSE IN GASTROINTESTINAL DISORDERS

\begin{tabular}{|c|c|c|c|c|c|c|c|c|c|c|c|c|c|c|}
\hline \multirow[t]{4}{*}{ Patient } & \multirow[t]{4}{*}{ Diagnosis } & \multirow{4}{*}{$\begin{array}{l}\text { Body } \\
\text { Weight } \\
\text { (kg.) }\end{array}$} & \multirow{4}{*}{$\begin{array}{l}\text { Secretin } \\
\text { Dose } \\
\text { (units/ } \\
\text { min.) }\end{array}$} & \multicolumn{6}{|c|}{ Response } & \multicolumn{4}{|c|}{ Mean Response (Average of Samples 2 and 3) } & \multirow{4}{*}{$\begin{array}{l}\text { Maxi- } \\
\text { mum } \\
\text { Bicarbon- } \\
\text { ate Con- } \\
\text { centra- } \\
\text { tion } \\
\text { (mEq./1.) }\end{array}$} \\
\hline & & & & \multirow{2}{*}{\multicolumn{3}{|c|}{$\begin{array}{l}\text { Volume (ml.I } \\
\text { min.): 10-Min. } \\
\text { Samples }\end{array}$}} & \multirow{2}{*}{\multicolumn{3}{|c|}{$\begin{array}{l}\text { Bicarbonate } \\
\text { ( } \mu \text { Eq./min.): } 10 \text { - } \\
\text { Min. Samples }\end{array}$}} & \multicolumn{2}{|l|}{ Volume } & \multicolumn{2}{|l|}{ Bicarbonate } & \\
\hline & & & & & & & & & & \multirow[t]{2}{*}{ ml./min. } & \multirow[t]{2}{*}{$\mu l . / \mathrm{kg} . / \mathrm{min}}$. & \multirow[t]{2}{*}{$\mu E q . /$ min. } & \multirow{2}{*}{$\begin{array}{l}\mu E q . \mid k g . / \\
\text { min. }\end{array}$} & \\
\hline & & & & 1 & 2 & 3 & 1 & 2 & 3 & & & & & \\
\hline M.B. & $\begin{array}{l}\text { After } \\
\text { cholecystectomy }\end{array}$ & $61 \cdot 2$ & $\begin{array}{r}2 \\
4 \\
8 \\
12 \\
15\end{array}$ & $\begin{array}{l}2.0 \\
3.0 \\
2.6 \\
4.1 \\
4.7\end{array}$ & $\begin{array}{l}2.9 \\
3.8 \\
3.5 \\
5.2 \\
5.6\end{array}$ & $\begin{array}{l}3 \cdot 3 \\
2 \cdot 9 \\
3 \cdot 3 \\
4 \cdot 0 \\
-\end{array}$ & $\begin{array}{l}210 \\
381 \\
304 \\
442 \\
447\end{array}$ & $\begin{array}{l}345 \\
459 \\
409 \\
546 \\
480\end{array}$ & $\begin{array}{l}398 \\
362 \\
300 \\
400 \\
-\end{array}$ & $\begin{array}{l}3 \cdot 1 \\
3 \cdot 3 \\
3 \cdot 4 \\
4 \cdot 6 \\
5 \cdot 6\end{array}$ & $\begin{array}{l}51 \\
54 \\
55 \\
75 \\
91\end{array}$ & $\begin{array}{l}371 \\
410 \\
354 \\
473 \\
480\end{array}$ & $\begin{array}{l}6.06 \\
6.70 \\
5 \cdot 78 \\
7 \cdot 73 \\
7.84\end{array}$ & $\begin{array}{r}121 \\
127 \\
117 \\
108 \\
95\end{array}$ \\
\hline M.L. & $\begin{array}{l}\text { After } \\
\text { cholecystectomy }\end{array}$ & $48 \cdot 6$ & $\begin{array}{l}4^{1} \\
6^{1} \\
8^{1}\end{array}$ & $\begin{array}{l}3 \cdot 8 \\
3 \cdot 4 \\
4 \cdot 6\end{array}$ & $\begin{array}{l}3.7 \\
4.5 \\
4.5\end{array}$ & $\begin{array}{l}3.9 \\
4 \cdot 5 \\
4 \cdot 5\end{array}$ & $\begin{array}{l}296 \\
391 \\
463\end{array}$ & $\begin{array}{l}385 \\
477 \\
436\end{array}$ & $\begin{array}{l}378 \\
441 \\
405\end{array}$ & $\begin{array}{l}3 \cdot 8 \\
4 \cdot 5 \\
4 \cdot 5\end{array}$ & $\begin{array}{l}78 \\
92 \\
92\end{array}$ & $\begin{array}{l}381 \\
459 \\
420\end{array}$ & $\begin{array}{l}7 \cdot 84 \\
9 \cdot 44 \\
8 \cdot 64\end{array}$ & $\begin{array}{l}104 \\
115 \\
103\end{array}$ \\
\hline L.M. & $\begin{array}{l}\text { Chronic } \\
\text { pancreatitis }\end{array}$ & $48 \cdot 6$ & $\begin{array}{l}2 \\
4 \\
8 \\
2^{1}\end{array}$ & $\begin{array}{l}0.1 \\
0.1 \\
0 \cdot 1 \\
1.6\end{array}$ & $\begin{array}{ll}0.1 & 0 \\
0.5 & 0 \\
0.3 & 0 \\
2.3 & 2\end{array}$ & $\begin{array}{l}0 \cdot 1 \\
0 \cdot 3 \\
0 \cdot 3 \\
2 \cdot 5\end{array}$ & $\begin{array}{r}1 \\
1 \\
1 \\
62\end{array}$ & $\begin{array}{r}1 \\
21 \\
7 \\
82\end{array}$ & $\begin{array}{r}1 \\
9 \\
9 \\
88\end{array}$ & $\begin{array}{l}0 \cdot 1 \\
0 \cdot 4 \\
0 \cdot 3 \\
2 \cdot 4\end{array}$ & $\begin{array}{r}2 \\
8 \\
6 \\
49\end{array}$ & $\begin{array}{r}1 \\
15 \\
8 \\
85\end{array}$ & $\begin{array}{l}0.02 \\
0 \cdot 31 \\
0 \cdot 16 \\
1.75\end{array}$ & $\begin{array}{l}13 \\
39 \\
31 \\
35\end{array}$ \\
\hline H.E. & $\begin{array}{l}\text { Chronic } \\
\text { pancreatitis }\end{array}$ & $56 \cdot 7$ & $\begin{array}{l}2 \\
6 \\
6^{1}\end{array}$ & $\begin{array}{l}0.3 \\
0.2 \\
2 \cdot 5\end{array}$ & $\begin{array}{l}0 \cdot 3 \\
0 \cdot 2 \\
1 \cdot 5\end{array}$ & $\begin{array}{r}0 \\
0 \cdot 6 \\
1.5\end{array}$ & $\begin{array}{r}0 \\
2 \\
77\end{array}$ & $\begin{array}{r}4 \\
2 \\
51\end{array}$ & $\begin{array}{r}0 \\
9 \\
51\end{array}$ & $\begin{array}{l}0 \cdot 1 \\
0 \cdot 4 \\
1 \cdot 5\end{array}$ & $\begin{array}{r}2 \\
7 \\
26\end{array}$ & $\begin{array}{r}2 \\
5 \\
51\end{array}$ & $\begin{array}{l}0.03 \\
0.09 \\
0.90\end{array}$ & $\begin{array}{l}12 \\
16 \\
34\end{array}$ \\
\hline L.S. & $\begin{array}{l}\text { Chronic } \\
\text { pancreatitis }\end{array}$ & $73 \cdot 3$ & $\begin{array}{l}1 \\
2 \\
3 \\
4\end{array}$ & $\begin{array}{r}0 \\
1.2 \\
0 \cdot 5 \\
1 \cdot 3\end{array}$ & $\begin{array}{l}0.8 \\
1.4 \\
0.9 \\
0.3\end{array}$ & $\begin{array}{l}0 \cdot 6 \\
0 \cdot 6 \\
0 \cdot 3 \\
0.9\end{array}$ & $\begin{array}{r}0 \\
0 \\
3 \\
26\end{array}$ & $\begin{array}{r}13 \\
0 \\
5 \\
2\end{array}$ & $\begin{array}{r}2 \\
10 \\
5 \\
13\end{array}$ & $\begin{array}{l}0.7 \\
1.0 \\
0.6 \\
0.6\end{array}$ & $\begin{array}{r}10 \\
14 \\
8 \\
8\end{array}$ & $\begin{array}{l}7 \\
5 \\
5 \\
7\end{array}$ & $\begin{array}{l}0 \cdot 10 \\
0 \cdot 07 \\
0 \cdot 07 \\
0 \cdot 10\end{array}$ & $\begin{array}{l}16 \\
17 \\
14 \\
20\end{array}$ \\
\hline C.A. & $\begin{array}{l}\text { Chronic } \\
\text { pancreatitis }\end{array}$ & $94 \cdot 5$ & $\begin{array}{l}1 \\
2 \\
4 \\
8\end{array}$ & $\begin{array}{l}1.0 \\
1.9 \\
1.3 \\
4.5\end{array}$ & $\begin{array}{l}3.7 \\
2.2 \\
3.3 \\
3.7\end{array}$ & $\begin{array}{l}2 \cdot 1 \\
1 \cdot 8 \\
4 \cdot 2 \\
3 \cdot 8\end{array}$ & $\begin{array}{r}37 \\
144 \\
95 \\
418\end{array}$ & $\begin{array}{l}241 \\
178 \\
304 \\
322\end{array}$ & $\begin{array}{l}168 \\
151 \\
404 \\
335\end{array}$ & $\begin{array}{l}2.9 \\
2.0 \\
3.7 \\
3.7\end{array}$ & $\begin{array}{l}31 \\
21 \\
39 \\
39\end{array}$ & $\begin{array}{l}204 \\
164 \\
354 \\
328\end{array}$ & $\begin{array}{l}2 \cdot 16 \\
1 \cdot 73 \\
3.74 \\
3.47\end{array}$ & $\begin{array}{l}80 \\
84 \\
96 \\
93\end{array}$ \\
\hline М.H. & $\begin{array}{l}\text { Unproved } \\
\text { chronic } \\
\text { pancreatitis }\end{array}$ & $48 \cdot 1$ & $\begin{array}{r}2 \\
4 \\
6 \\
8 \\
12\end{array}$ & $\begin{array}{l}0.2 \\
0.4 \\
0.3 \\
2.1 \\
3.4\end{array}$ & $\begin{array}{l}0 \cdot 5 \\
0 \cdot 3 \\
3 \cdot 4 \\
2 \cdot 5 \\
2 \cdot 8\end{array}$ & $\begin{array}{l}0 \cdot 3 \\
0 \cdot 5 \\
2 \cdot 8 \\
2 \cdot 4 \\
2 \cdot 6\end{array}$ & $\begin{array}{r}4 \\
10 \\
16 \\
212 \\
221\end{array}$ & $\begin{array}{r}4 \\
12 \\
312 \\
252 \\
235\end{array}$ & $\begin{array}{r}12 \\
12 \\
257 \\
238 \\
244\end{array}$ & $\begin{array}{l}0 \cdot 4 \\
0 \cdot 4 \\
3 \cdot 1 \\
2 \cdot 4 \\
2 \cdot 7\end{array}$ & $\begin{array}{r}8 \\
8 \\
64 \\
52 \\
56\end{array}$ & $\begin{array}{r}8 \\
12 \\
284 \\
245 \\
239\end{array}$ & $\begin{array}{l}0 \cdot 17 \\
0 \cdot 25 \\
5 \cdot 91 \\
5 \cdot 10 \\
4.97\end{array}$ & $\begin{array}{r}13 \\
35 \\
92 \\
101 \\
94\end{array}$ \\
\hline D.C. & $\begin{array}{l}\text { Post-acute } \\
\text { pancreatitis }\end{array}$ & $58 \cdot 5$ & $\begin{array}{l}2 \\
6 \\
8 \\
6^{1}\end{array}$ & $\begin{array}{l}1 \cdot 4 \\
3 \cdot 1 \\
2.6 \\
5.0\end{array}$ & $\begin{array}{l}1.1 \\
5.0 \\
5.7 \\
6.8\end{array}$ & $\begin{array}{l}1.5 \\
3 \cdot 6 \\
5 \cdot 8 \\
6.5\end{array}$ & $\begin{array}{l}144 \\
363 \\
278 \\
540\end{array}$ & $\begin{array}{l}110 \\
505 \\
616 \\
666\end{array}$ & $\begin{array}{l}166 \\
364 \\
621 \\
617\end{array}$ & $\begin{array}{l}1 \cdot 3 \\
4 \cdot 3 \\
5 \cdot 7 \\
6 \cdot 6\end{array}$ & $\begin{array}{r}22 \\
73 \\
97 \\
112\end{array}$ & $\begin{array}{l}138 \\
434 \\
618 \\
641\end{array}$ & $\begin{array}{r}2.36 \\
7.42 \\
10.56 \\
10.96\end{array}$ & $\begin{array}{l}111 \\
117 \\
108 \\
108\end{array}$ \\
\hline B.G. & $\begin{array}{l}\text { Adult coeliac } \\
\text { disease }\end{array}$ & $59 \cdot 3$ & $\begin{array}{l}2 \\
6 \\
8 \\
6^{1}\end{array}$ & $\begin{array}{l}2.3 \\
1.0 \\
1.3 \\
6.0\end{array}$ & $\begin{array}{l}1.9 \\
1.6 \\
3.2 \\
4.2\end{array}$ & $\begin{array}{l}0.6 \\
1.6 \\
3.0 \\
5.0\end{array}$ & $\begin{array}{r}2 \\
53 \\
60 \\
342\end{array}$ & $\begin{array}{r}13 \\
136 \\
240 \\
328\end{array}$ & $\begin{array}{r}11 \\
144 \\
290 \\
385\end{array}$ & $\begin{array}{l}1 \cdot 2 \\
1 \cdot 6 \\
3 \cdot 1 \\
4 \cdot 6\end{array}$ & $\begin{array}{l}20 \\
27 \\
52 \\
78\end{array}$ & $\begin{array}{r}12 \\
140 \\
265 \\
356\end{array}$ & $\begin{array}{l}0.20 \\
2.36 \\
4.46 \\
6.00\end{array}$ & $\begin{array}{l}19 \\
90 \\
97 \\
78\end{array}$ \\
\hline C.G. & $\begin{array}{l}\text { Adult coeliac } \\
\text { disease }\end{array}$ & $55 \cdot 8$ & $\begin{array}{l}2 \\
4 \\
8 \\
2^{1}\end{array}$ & $\begin{array}{l}0.4 \\
0.7 \\
1.9 \\
2.5\end{array}$ & $\begin{array}{l}0.6 \\
0.7 \\
1.9 \\
3.2\end{array}$ & $\begin{array}{l}0.9 \\
1.9 \\
2.0 \\
4.4\end{array}$ & $\begin{array}{r}18 \\
72 \\
216 \\
-\end{array}$ & $\begin{array}{r}28 \\
72 \\
206 \\
224\end{array}$ & $\begin{array}{r}79 \\
186 \\
154 \\
277\end{array}$ & $\begin{array}{l}0.7 \\
1.3 \\
1.9 \\
3.8\end{array}$ & $\begin{array}{l}12 \\
23 \\
34 \\
68\end{array}$ & $\begin{array}{r}53 \\
129 \\
180 \\
250\end{array}$ & $\begin{array}{l}0.95 \\
2 \cdot 31 \\
3 \cdot 23 \\
4 \cdot 48\end{array}$ & $\begin{array}{r}86 \\
98 \\
111 \\
69\end{array}$ \\
\hline R.M. & Diabetes mellitus & $79 \cdot 6$ & $\begin{array}{l}2 \\
4 \\
6 \\
8\end{array}$ & $\begin{array}{l}0 \cdot 5 \\
3 \cdot 6 \\
3 \cdot 2 \\
4 \cdot 2\end{array}$ & $\begin{array}{l}2.0 \\
3 \cdot 1 \\
3 \cdot 0 \\
2.6\end{array}$ & $\begin{array}{l}2 \cdot 1 \\
2 \cdot 3 \\
3 \cdot 0 \\
3 \cdot 3\end{array}$ & $\begin{array}{r}11 \\
291 \\
313 \\
420\end{array}$ & $\begin{array}{r}84 \\
202 \\
306 \\
222\end{array}$ & $\begin{array}{l}189 \\
205 \\
306 \\
346\end{array}$ & $\begin{array}{l}2.0 \\
2.7 \\
3.0 \\
2.9\end{array}$ & $\begin{array}{l}25 \\
34 \\
38 \\
36\end{array}$ & $\begin{array}{l}136 \\
203 \\
306 \\
284\end{array}$ & $\begin{array}{l}1.71 \\
2.55 \\
3.84 \\
3.56\end{array}$ & $\begin{array}{r}90 \\
89 \\
102 \\
105\end{array}$ \\
\hline D.T. & $\begin{array}{l}\text { Idiopathic } \\
\text { steatorrhoea }\end{array}$ & $41 \cdot 8$ & $\begin{array}{l}2 \\
4 \\
6\end{array}$ & $\begin{array}{l}2 \cdot 0 \\
3 \cdot 4 \\
5 \cdot 2\end{array}$ & $\begin{array}{l}2 \cdot 4 \\
3 \cdot 8 \\
6 \cdot 0\end{array}$ & $\begin{array}{l}2 \cdot 0 \\
6 \cdot 0 \\
6 \cdot 5\end{array}$ & $\begin{array}{l}110 \\
357 \\
527\end{array}$ & $\begin{array}{l}230 \\
338 \\
570\end{array}$ & $\begin{array}{l}202 \\
606 \\
605\end{array}$ & $\begin{array}{l}2 \cdot 2 \\
4 \cdot 9 \\
6 \cdot 2\end{array}$ & $\begin{array}{r}52 \\
117 \\
148\end{array}$ & $\begin{array}{l}216 \\
472 \\
587\end{array}$ & $\begin{array}{r}5 \cdot 16 \\
11 \cdot 30 \\
14 \cdot 00\end{array}$ & $\begin{array}{l}101 \\
105 \\
101\end{array}$ \\
\hline J.T. & $\begin{array}{l}\text { Diarrhoea, } \\
\text { hyperchlorhydria }\end{array}$ & $51 \cdot 7$ & $\begin{array}{r}2 \\
4 \\
8 \\
12 \\
15\end{array}$ & $\begin{array}{l}1.7 \\
1.4 \\
2.1 \\
3.5 \\
6.0\end{array}$ & $\begin{array}{l}3 \cdot 5 \\
2 \cdot 8 \\
2 \cdot 7 \\
5 \cdot 2 \\
8 \cdot 7\end{array}$ & $\begin{array}{l}3.6 \\
2.1 \\
4.0 \\
7.0 \\
-\end{array}$ & $\begin{array}{r}37 \\
62 \\
65 \\
404 \\
635\end{array}$ & $\begin{array}{r}170 \\
90 \\
223 \\
589 \\
956\end{array}$ & $\begin{array}{l}184 \\
128 \\
448 \\
834 \\
--\end{array}$ & $\begin{array}{l}3 \cdot 5 \\
2 \cdot 4 \\
3 \cdot 3 \\
6 \cdot 1 \\
8 \cdot 7\end{array}$ & $\begin{array}{r}68 \\
46 \\
64 \\
118 \\
168\end{array}$ & $\begin{array}{l}177 \\
109 \\
335 \\
711 \\
956\end{array}$ & $\begin{array}{r}3.42 \\
2.11 \\
6.48 \\
13.76 \\
18.50\end{array}$ & $\begin{array}{r}52 \\
61 \\
112 \\
116 \\
110\end{array}$ \\
\hline
\end{tabular}

'Infusion of Cecekin (cholecystokinin-pancreozymin) with secretin. 
(a)

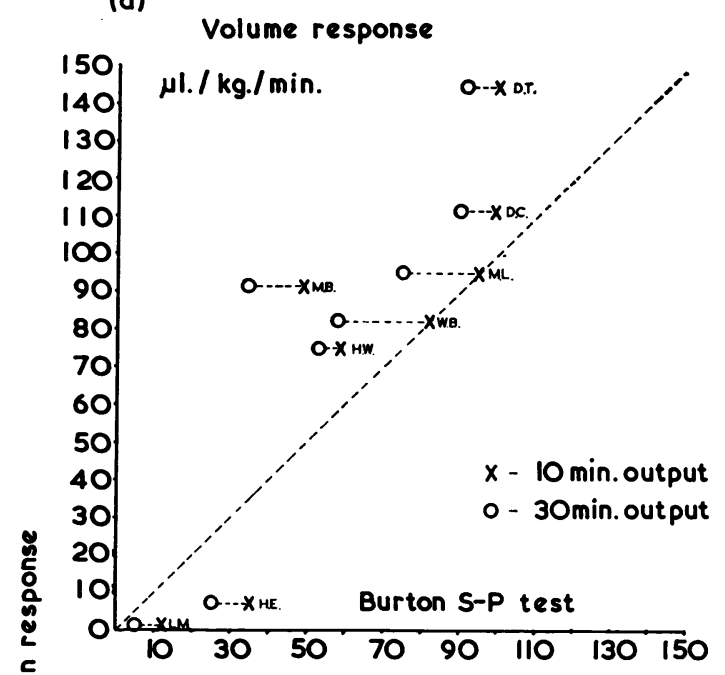

(b)

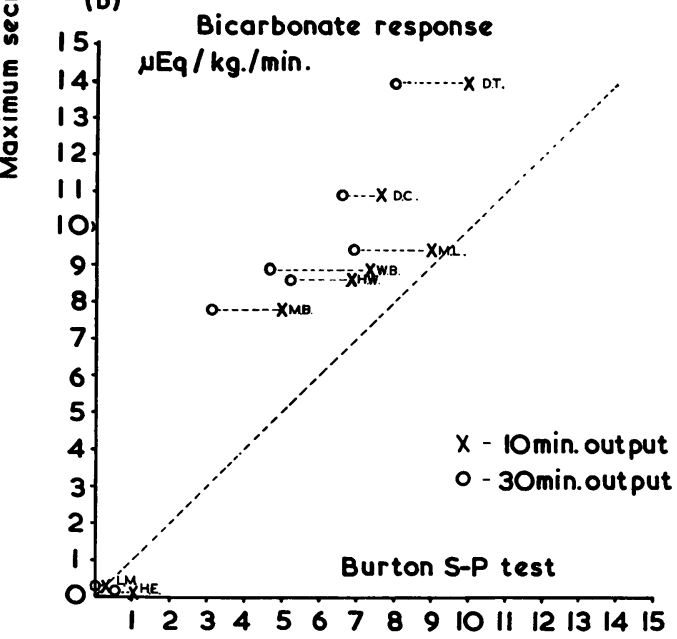

FIG. 5. A comparison of (a) the volume response and (b) the bicarbonate response obtained by the Burton test and maximum secretin infusion. mum secretory response and body weight in dogs may be attributable to the small differences in weight of the animals which were studied.

Comparison between results obtained by the Burton test and the maximum infusion of secretin show that the latter procedure gave the greatest volume and bicarbonate response. The coefficients of variation for volume and maximum bicarbonate concentration (Table V) were similar to those reported by authors using standard secretin procedures (Dreiling and Janowitz, 1962; Sun, 1963; and Bank, Marks, Moshal, Efron, and Silber, 1963). However, contrary to the experience of Burton et al. (1960) and Dreiling and Janowitz (1962) the coefficient of variation for maximum volume and bicarbonate output was greatly reduced when the output was related to body weight (Table V).

The values for maximum volume and bicarbonate output in this study were similar to the values obtained by Hartley, Gambill, and Summerskill (1965) using a single injection of secretin administered intravenously over a period of 2 to 3 minutes. The coefficients of variation of their responses were, however, much greater (peak volume output $48 \%$, peak bicarbonate output $/ \mathrm{kg}$. body weight $24 \%$ ) than when a constant infusion of secretin was used.

The results obtained on patients with various gastrointestinal diseases demonstrate the application of the maximum infusion procedure, which promises to be a valuable method for the investigation of pancreatic dysfunction. The technique is free from side effects and should take no longer to perform than a standard secretin test.

\section{SUMMARY}

The secretory response of the human pancreas to continuous intravenous infusion of secretin was measured in normal subjects and in patients suspected of having pancreatic disease.

The maximum volume and bicarbonate output was related to body wieght.

\section{TABLE V}

ANALYSIS OF MAXIMUM VOLUME AND BICARBONATE RESPONSE AND MAXIMUM BICARBONATE CONCENTRATION IN SEVEN NORMAL SUBJECTS

\begin{tabular}{|c|c|c|c|c|c|}
\hline & Mean & S.D. $(n=6)$ & \multicolumn{2}{|c|}{$\begin{array}{l}95 \% \text { Confidence Limits of a Single Observa- } \\
\text { tion about the Mean }(n=6 t=2.45)\end{array}$} & $\begin{array}{l}\text { Coefficient } \\
\text { of Variation }\end{array}$ \\
\hline $\begin{array}{l}\text { Maximum volume response } Y_{1} \\
\text { (a) } \mathrm{ml} . / \mathrm{min} . \\
\text { (b) } \mu \mathrm{l} . / \mathrm{kg} . / \mathrm{min} .\end{array}$ & $\begin{array}{r}4 \cdot 60 \\
74 \cdot 71\end{array}$ & $\begin{array}{l}1 \cdot 03 \\
6 \cdot 21\end{array}$ & $\begin{array}{r}2 \cdot 1 \\
59 \cdot 5\end{array}$ & $\begin{array}{r}7 \cdot 1 \\
89 \cdot 9\end{array}$ & $\begin{array}{r}22 \cdot 3 \% \\
8 \cdot 3 \%\end{array}$ \\
\hline $\begin{array}{l}\text { Maximum bicarbonate response } Y_{2} \\
\text { (a) } \mu E_{q} . / \mathrm{min} . \\
\text { (b) } \mu E_{q} / \mathrm{kg} . / \mathrm{min} \text {. }\end{array}$ & $\begin{array}{r}520 \cdot 3 \\
8.48\end{array}$ & $\begin{array}{r}125 \cdot 0 \\
1 \cdot 15\end{array}$ & $\begin{array}{r}214 \\
5 \cdot 7\end{array}$ & $\begin{array}{l}826 \\
11 \cdot 3\end{array}$ & $\begin{array}{l}24.0 \% \\
13.6 \%\end{array}$ \\
\hline $\begin{array}{l}\text { Maximum bicarbonate concentration } \\
\text { mEq./1. }\end{array}$ & $123 \cdot 7$ & 6.8 & 107 & 140 & $5.5 \%$ \\
\hline
\end{tabular}


A comparison with a standard secretin test procedure showed that maximum infusion of secretin may be a useful method for investigating pancreatic dysfunction.

We are most grateful to Dr. Robert Gaddie for advice and criticism during the course of this work.

\section{REFERENCES}

Bank, S., Marks, I. N., Moshal, M. G., Efron, G., and Silber, R. (1963) The pancreatic-function test method and normal values. $S$. Afr. med. J., 37, 1061-1066.

Baron, J. H., Perrier, C. V., Janowitz, H. D., and Dreiling, D. A. (1963) Maximum alkaline (bicarbonate) output of the dog pancreas. Amer. J. Physiol., 204, 251-256.

Bertalanffy, L. V. (1960). Principles and theory of growth in Fundamental Aspects of Normal and Malignant Growth, edited by W. Nowinski., pp. 137-259. Elsevier, Amsterdam and London.

Burton, P., Evans, D. G., Harper, A. A., Howat, H. T., Oleesky, S. Scott, J. E., and Varley, H. (1960). A test of pancreatic function in man based on the analysis of duodenal contents after administration of Secretin-Pancreozymin. Gut., 1, 111-124.

Card, W. I., and Marks, I. N. (1960). The relationship between the acid output of the stomach following 'maximal' histamine stimulation and the parietal cell mass. Clin. Sci., 19, 147-163.

Chey, W. Y., Shay, H., and Shuman, C. R. (1963). External pancreatic secretion in diabetes mellitus. Ann. intern. Med., 59, 812-821.

Dreiling, D. A., and Janowitz, H. D. (1962). The measurement of pancreatic secretory function. In Ciba Foundation Symposium on the Exocrine Pancreas, edited by A. V. S. de Reuck and M. P. Cameron, p.p. 225-258. Churchill, London.
Hansky, J., Tiscornia, O. M., Dreiling, D. A., and Janowitz, H. D. (1963). Relationship between maximal secretory output and weight of the pancreas in the dog. Proc. Soc. exp. Biol. (N.Y.), $114,654-656$

-, 1 , (1964). Maximal secretory capacity of the canine pancreas in response to pancreazymin and secretin. Amer. J. Physiol., 206, 351-356.

Hartley, R. C., Gambill, E. E., and Summerskill, W. H. J. (1965). Pancreatic volume and bicarbonate output with augmented doses of secretin. Gastroenterology, 48, 312-317.

Jorpes, E., and Mutt, V. (1964). Gastrointestinal hormones. In The Hormones, vol. 4, p.p. 365-385, edited by G. Pincus, K. V. Thimann, and E. B. Astwood. Academic Press, New York.

Kay, A. W. (1953). Effect of large doses of histamine on gastric secretion of $\mathrm{HCl}$ : an augmented histamine test. Brit. med. J., 2, 77-80.

Lawrie, J. H., Smith, G. M. R., and Forrest, A. P. M. (1964). The histamine-infusion test. Lancet, 2, 270-273.

Marks, I. N. (1961). The augmented histamine test. Gastroenterology, 41, 599-603.

Northam, B. E., and Banwell, J. G. (1966). Automated analysis in the investigation of pancreatic function. Technicon European Symposium, Paris.

Payne, R. A., and Kay, A. W. (1962). The effect of vagotomy on the maximal acid sezretory response to histamine in man. Clin. Sci., 22, 373-382.

Pollard, H. M., Miller, L., and Brewer, W. A. (1943). The external secretion of the pancreas and diabetes mellitus. Amer. J. dig. Dis., 10, 20-23.

Sarles, H., Bauer, J. B., and Prezlin, G. (1965). Étude des injections répétées et des perfusions continues de sécrétine chez l'homme. Arch. Mal. Appar. dig., 54, 177-194.

Scheidt, R. A., and Gochman, N. (1964). An automated amyloclastic procedure for the determination of serum amylase. Technicon 4th International Symposium on Automation in Analytical Chemistry, London, 1964.

Sun, D. C. H. (1963). Normal values for pancreozymin-secretin test. Gastroenterology, 44, 602-606. 